\title{
Artificial ventilation, prolonged endotracheal intubation and tracheostomy in paediatric surgery
}

\author{
W. J. GLOVER \\ M.B., B.Ch., F.F.A.R.C.S., D.Obst.R.C.O.G. \\ Hospital for Sick Children, Great Ormond Street, London, W.C.1
}

THE young infant has much less respiratory reserve than older children and adults; for example, his resting oxygen consumption, per unit of body weight, is twice that of the adult, $7 \mathrm{ml} / \mathrm{kg} / \mathrm{min}$ compared with $3.5 \mathrm{ml} / \mathrm{kg} / \mathrm{min}$. The diameters of distal airways remain constant from birth until about 5 years of age and only then increase in size (Hogg et al., 1970). Consequently, infants and young children have a high peripheral airways resistance and a greater tendency to airways occlusion than older children.

The average newborn infant has about twice the surface for heat loss for each kilogram of tissue compared with the average adult (Cross, 1965). If the infant is placed in a cool environment his metabolism increases to attempt to maintain his body temperature. This increase in metabolism in turn increases his oxygen consumption and minute volume. Nursing an infant in a cool environment places an additional demand on the respiratory system. In an infant already in respiratory distress this could result in respiratory insufficiency. The importance of conserving heat in the care of ill infants is therefore of the utmost importance.

In infants, as in adults, the cause of respiratory insufficiency may be decreased respiratory movements or pulmonary complications or a combination of both. For the reasons given above, however, infants will reach a state of respiratory insufficiency more readily than older children.

\section{Essential features of a paediatric ventilator}

\section{(1) Tidal volume}

The tidal volume of an infant varies inversely with the respiratory rate and ranges from about 10 to 30 $\mathrm{ml}$ in patients of about $3.5 \mathrm{~kg}$. The ventilator must deliver volumes in this range to the patient. The volume indicated by a spirometer placed in the conventional position in the expiratory limb of a circuit does not represent the patient's minute volume. The figure obtained from the spirometer must be corrected to allow for compression of the gas in the patient circuit. Gas compressed in the circuit on inspiration will re-expand during expiration when the pressure falls and contribute with the expired gas from the patient to the reading on the spirometer (Glover, 1965). The volume of the circuit, the peak inspiratory pressure reached in the circuit, and the frequency of ventilation determine the size of this compressibility factor. The discrepancy between the patient's minute volume and spirometer reading will therefore be greatest in infants requiring high inspiratory pressures.

\section{(2) Pressure}

The inspiratory pressure required to ventilate the infant lung varies considerably. It will be less than $10 \mathrm{~cm} \mathrm{H}_{2} \mathrm{O}$ if the lungs are normal and it may be as high as $50 \mathrm{~cm} \mathrm{H}_{2} \mathrm{O}$ in infants with pulmonary oedema and bronchiolitis. It is therefore essential to be able to apply pressures of this order when the clinical condition requires it.

\section{(3) Accurate control of inspired oxygen}

In patients of all ages there is a danger of pulmonary damage if oxygen concentrations above $50 \%$ are administered for a number of days.

Young animals exposed to $100 \%$ oxygen at 1 atmosphere die in 72-96 hours. At autopsy the lungs are large, dark, heavy and liver-like in appearance. Man appears less susceptible and human volunteers showed a fall in pulmonary diffusion capacity after $30 \mathrm{hr}$ exposure to $98 \%$ oxygen at 1 atmosphere and a fall in vital capacity after $60 \mathrm{hr}$ exposure (Caldwell et al., 1966).

It is well known that high percentages of oxygen administered to premature infants may result in blindness due to retrolental fibroplasia. This complication may arise if the $\mathrm{PaO}_{2}$ is above $150 \mathrm{mmHg}$ in the retinal vessels. If the oxygen tension in the arterial blood is monitored from the umbilical artery in the immediate newborn period lower values will be obtained than exist in the vessels perfusing the eye and brain. This is due to shunting from right-toleft via the ductus arteriosus. Where a considerable right-to-left shunt exists, as in respiratory distress, a $\mathrm{PaO}_{2}$ of $60-90 \mathrm{mmHg}$ in the umbilical artery blood sample may be acceptable (Baum \& Tizard, 1970).

In the care of ill patients there should be no 
hesitation in raising the inspired oxygen concentration as much as is necessary to maintain acceptable arterial oxygen tensions. Retrolental fibroplasia will not occur unless the arterial oxygen tension is abnormally high and the danger of pulmonary damage from the high inspired oxygen concentration is not acute enough to outweigh the serious and immediate consequences of severe oxygen desaturation.

It is therefore essential to be able to control accurately the percentage oxygen delivered by a ventilator. The inspired oxygen concentration should in addition be checked regularly with an oxygen analyser. When oxygen-rich mixtures are administered it is also necessary to check the arterial oxygen tension.

\section{(4) Humidification}

Since the artificial airway (endotracheal tube or tracheostomy) by-passes the patient's humidifying mechanism, the nose, it is essential to humidify the inspired gas. This aspect is often overlooked in considering the merits of various ventilators. In infants it is of even greater importance because the inspissation of secretions resulting from the inspiration of dry gas readily blocks the narrow airways of the patient and also the lumen of the endotracheal or tracheostomy tube. We should try to achieve a relative humidity of $70 \%$ or more in order to maintain ciliary activity, as a low relative humidity has a retarding effect on ciliary motion (Dalhamn, 1956).

There are three methods of increasing the water content in the inspired air and only brief comments are made on them here:

(a) Standard humidification. This involves the heating of water over which gas is passed to the patient. If the water in the humidifier is kept at about $55^{\circ} \mathrm{C}$ then all vegetative organisms are killed. This eliminates an important source of proliferation of bacteria. The disadvantage of this method is that the temperature of the inspired gas falls as it passes along the inspiratory tubing to the patient. It is desirable to keep the temperature at the patient end of the tubing at approximately $35^{\circ} \mathrm{C}$ in young infants and $33^{\circ} \mathrm{C}$ in older children. In young infants humidified gas is a potent factor in maintaining body temperature as the patient does not have to produce water vapour from his respiratory tract and thereby lose heat.

(b) Compressed air nebulizer. This gives a variable droplet size and a high proportion of the droplets may be comparatively large. Droplets greater than $10 \mu$ are deposited in the upper trachea. The output from compressed air nebulizers is low even with high gas flow rates. With low gas flow rates as used in paediatric practice the output may be extremely small (Fenstermaker, 1970).

(c) Ultrasonic nebulizer. These are extremely effec- tive provided an artificial airway is in place. If the patient is breathing through his nose then neither ultrasonic nor compressed air nebulizers are effective because the nose filters out the droplets (Wolfsdorf, Swift \& Avery, 1969). When however an endotracheal or tracheostomy tube is in place, the output from the nebulizer must be carefully controlled as it is easy to overload an infant's lungs with fluid. Saline is more dangerous than water in this respect and consequently distilled water should be used (Modell et al., 1968).

An important disadvantage of both compressed air and ultrasonic nebulizers is the possible transmission of bacteria to the patient (Moffet \& Allan, 1967). Any device producing mists is liable to disseminate quantities of bacteria. The smaller particles produced by ultrasonic nebulizers are particularly dangerous as they penetrate deeply into the lungs where pulmonary clearance mechanisms may not be efficient.

Few ventilators will meet all four criteria described above. The clinician in choosing a machine must bear in mind the nature of the clinical problems with which he will be confronted in order that he may make the best choice for his particular purpose.

\section{Management}

Continuous nursing supervision is essential in this work. The objective in mechanical ventilation is to maintain the patient's arterial $\mathrm{PO}_{2}$ and $\mathrm{PCO}_{2}$ near physiological levels and this is most easily achieved by taking complete control of the patient's respiration.

The use of patient-triggered ventilators would seem attractive but in practice in infants breathing rapidly it can be extremely difficult to achieve satisfactory patient-triggering. Under-ventilation will then occur.

If one ventilates children satisfactorily so that the $\mathrm{PaO}_{2}$ and $\mathrm{PaCO}_{2}$ are approximately normal then the patient will follow the cycling of the ventilator and cease making his own efforts. There are three exceptions to this generalization:

(1) If the patient is in pain following surgery then an analgesic such as morphine is required to gain control.

(2) If an infant is hungry then a feed is required to establish control.

(3) If there are large right-to-left shunts causing arterial oxygen desaturation as in respiratory distress syndrome or pneumonia then mechanical ventilation will not correct the blood gases and the patient tends to 'fight the ventilator'. When this occurs respiratory depressants such as morphine or muscle relaxants such as curare are required.

In general however, control of respiration can be accomplished without resorting to drugs. 
The avoidance of muscle relaxant drugs in the management of these patients ensures that a patient who is being under-ventilated will attempt to breathe spontaneously. When a patient ceases to follow the ventilator one should assume that he is not being adequately ventilated until proved otherwise. There may be several reasons for underventilation; secretions in the airway, collapse of a lobe of a lung, pneumothorax, an accidental change in the settings of the ventilator, or a fault in the ventilator. All these possibilities should be checked before resorting to drugs.

When putting an infant on a ventilator one must consider the rate, volume and pressures to be used.

\section{Rate}

Infants in respiratory distress have a very high respiratory rate because it is easier for the infant to increase his rate of respiration than his depth of respiration. It is not necessary in mechanical ventilation to follow this pattern as rates of ventilation near the physiological range are satisfactory, i.e. $30-40 / \mathrm{min}$. It may be that this slower rate of ventilation may result in more even distribution of gas, especially in diseased lungs, but this is difficult to prove in clinical practice.

\section{Minute volume}

The difficulties encountered in attempting to study the normal ventilation in the newborn have been stated in detail by Cross (1965). The effect of a cold environment in causing an increase in ventilation in the newborn has already been mentioned.

There are probably wide variations from infant to infant. A useful average figure to take is $600 \mathrm{ml}$ as the minute volume with a tidal volume of $15 \mathrm{ml}$ at a respiratory rate of $40 / \mathrm{min}$ in an infant weighing $3.5 \mathrm{~kg}$. It must be appreciated that this is a mean figure.

Having decided on a particular minute volume, the measurement of the minute volume produced by the ventilator presents problems. The errors involved in using a spirometer have been described earlier. Accurate measurements of tidal and minute volumes can be obtained by using a pneumotachograph which measures flow and this can be integrated with time to measure volume. However, to do this an airtight fit is required around the endotracheal or tracheostomy tube and this is not advisable in clinical practice for the reasons given later. In addition, such instruments require careful calibration and are fairly difficult to use in everyday practice.

In clinical practice it is more useful to assess the patient's colour and the adequacy of movement of the chest wall as one would do in anaesthesia. Blood gas analysis can then be done to confirm the adequacy of ventilation. Provided the peripheral circulation is good, a capillary sample from a heel prick is satisfactory. When the peripheral circulation is poor, a peripheral arterial sample should be taken if the umbilical artery is not available.

\section{Pressure}

As stated earlier, the pressure required to ventilate the lung will vary considerably depending on the airway resistance and lung compliance.

The correct pressure in a particular patient is the pressure which will produce adequate tidal volumes. High pressures are not dangerous provided they are necessary because the pressure is largely dissipated in overcoming the high airway resistance. They would be dangerous if applied to the normal lung because of the likelihood of producing a pneumothorax or of impairing pulmonary blood flow.

\section{Discontinuation of mechanical ventilation}

Once the respiratory insufficiency which created the need for mechanical ventilation is no longer present there is generally no difficulty in removing the patient from the ventilator. A useful method is to try the patient off the ventilator on an oxygenenriched mixture for progressively longer periods until he is completely off. This may be achieved about $24 \mathrm{hr}$ after commencing withdrawal. A patient who is unable to tolerate near-normal inspired oxygen concentrations while on the ventilator is unlikely to be able to do without the ventilator.

\section{Complications}

The means of minimizing most complications have already been dealt with. If a pneumothorax occurs, a chest drain connected to an under-water seal should be inserted and positive pressure ventilation continued. This complication should be very uncommon if correct pressures are used.

The danger of cross-infection by the ventilator can be avoided by sterilizing the patient circuit after use.

In infants with a very high airway resistance it is necessary, as already described, to use pressures of $40 \mathrm{~cm} \mathrm{H}_{2} \mathrm{O}$ or more to inflate the lungs. In such patients the lungs become over-inflated because there is insufficient motive force to expel the air through the narrow airways during expiration (Barnes et al., 1969). Discontinuing mechanical ventilation in these patients often takes several weeks. This complication is only seen in infants with a high airway resistance and has not been observed apart from this.

\section{Prolonged endotracheal intubation and tracheostomy}

Positive pressure ventilation of the lungs requires the insertion of an artificial airway, either an endotracheal tube or a tracheostomy tube. The precise role of endotracheal intubation or tracheostomy in 
the care of these patients has aroused considerable controversy over the past 9 years.

The most commonly used polymer for endotracheal or tracheostomy tubes is polyvinyl chloride (PVC). When formed by itself into a device it is hard, brittle, inflexible and translucent. The heat used to form the device tends to cause thermal degradation. This is shown by a yellowing of the material.

In order to confer flexibility, various materials known as plasticizers are used and to retard thermal degradation various materials known as stabilizers are used (Guess, 1970).

Guess \& Stetson (1968) identified a toxic substance which leached out of some PVC materials when these were inserted in rabbit muscle. Necrosis occurred in the muscle around the plastic insert. This substance was an organotin compound which is one of the most widely used stabilizers. Most manufacturers have now ceased using organotin compounds for this purpose and substituted epoxydized soya oil as a stabilizer. Users must however realize that there are various additives in PVC tubes and that not all manufacturers' products contain the same substances.

In the case of tracheostomy or endotracheal tubes no satisfactory test has yet been published that reveals the subtle effects of these devices upon mucous tissues (Guess, 1970).

\section{Prolonged endotracheal intubation}

\section{Management and complications}

Nasotracheal intubation is generally preferred to oral intubation as it is easier to fix the nasal tube to the face. There is then less danger of the ventilator tubing dislodging the endotracheal tube.

In all patients, every complication of an artificial airway, whether it is an endotracheal or a tracheostomy tube, must be regarded as extremely serious. Consequently, it is of the utmost importance to observe the following details:

(1) Incorrect length of endotracheal tube. As the infant's head may be flexed or extended in the dayto-day care of the patient, there will be some movement of the endotracheal tube up and down the trachea. The length of the tube must therefore be determined fairly precisely to avoid, on the one hand, endobronchial intubation and, on the other, dislodgement of the tube from the trachea. A useful method is to insert the tube via the nose until the tip of the tube is almost at the glottis; the tube is then cut to allow an appropriate length to pass the larynx and reach the mid-point of the trachea.

(2) Kinking. The most likely site at which kinking occurs is when a redundant length of tube protrudes from the nostril. The comparatively heavy connections to a ventilator will cause it to kink at this point and this is a further reason for ensuring that the tube is the correct length.

(3) Erosion of ala or septum of nose. If the tube presses on the edge of the nostril or septum, erosion can appear within $24 \mathrm{hr}$. If the pressure is not relieved, erosion through the lateral wall of the nose or the septum can occur in a few days. Considerable care is required in avoiding this complication. The tube should be fixed in such a way that it protrudes downwards from the nostril and therefore does not press on the edge of the nostril.

(4) Blockage of the tube. This is a serious and common occurrence if care is not taken to ensure good humidification and regular aspiration of the tube. Instruction must be given to the nurse to ensure that she passes the suction catheter all the way through the tube to the trachea.

(5) Sub-glottic oedema and sub-glottic stenosis. This has been the most serious of all complications and in the early reports it appeared in almost all series. The incidence was low, $5 \%$ in some series, and this encouraged the view that this complication was preventable. A widely held current view is that the tubes used in a few cases in the earlier series were too large.

The endotracheal tube, unlike tubes in the urethra or oesophagus must pass a non-distensible area at the level of the cricoid cartilage. Prolonged pressure on the mucosa at this level leads to ischaemia, oedema and ultimately, fibrosis. Treatment of a severe stenosis is extremely difficult and a long-term tracheostomy is usually the unfortunate result.

In paediatric anaesthetic practice one normally uses the largest tube which will easily pass the larynx in order to have an airtight fit. While this is satisfactory for periods of a few hours, it is probable that in prolonged use (over $24 \mathrm{hr}$ ) some degree of oedema and ischaemia occurs. Stocks (1970) therefore recommends that the correct size of tube is $0.5 \mathrm{~mm}$ internal diameter less than the size which gives an airtight fit. A useful guide for the inexperienced is that when positive pressure is applied to the tube to inflate the lungs an audible leak occurs around the tube at laryngeal level. It is this leak which leads to difficulty in using the pneumotachograph referred to earlier.

\section{Tracheostomy}

In the past decade, while experience has built up with prolonged endotracheal intubation, the management of tracheostomy has improved considerably. Complications such as death during the operative procedure, blockage or dislodgement of the tracheostomy tube, pneumothorax, tracheal stenosis and delayed decannulation are largely preventable (Fearon, 1962: Holinger, Brown \& Maurizi, 1965: Aberdeen, 1965: Glover, 1970). Attention to detail is as important as in prolonged endotracheal 
intubation because complications, which are on the whole preventable, are serious.

\section{Technique}

Anaesthesia. Tracheostomy in young children used to be performed as an emergency, often under local anaesthesia, and quite often in the ward rather than in the operating theatre. This resulted in a high operative mortality from asphyxia. Today the normal practice is to administer a general anaesthetic via an endotracheal tube. This ensures a good airway and a well-oxygenated motionless patient so that a planned operation can take place without haste.

In an emergency situation an endotracheal tube can be passed in the ward and the tracheostomy can then take place as a planned procedure in the operating theatre.

Surgical aspects. There are two points to observe:

(1) The first tracheal ring should be left intact as division of it can lead to perichondritis and subglottic stenosis (Watts, 1963).

(2) No cartilage should be excised in the infant trachea. If cartilage is excised it does not regenerate and healing is by fibrous tissue with consequent narrowing (Watts, 1963).

These points can be met by making a vertical incision in the mid-line of the trachea through the third and fourth (Holinger, et al., 1965) or third, fourth and fifth tracheal rings (Aberdeen, 1968). After insertion of the tube it is important to flex the neck fully when tying the tapes as the distance from the tracheostomy to the posterior aspect of the neck is minimal in this position. Failure to do this will result in the tapes being comparatively loose with consequent danger of dislodgement of the tube postoperatively.

Tracheostomy tube. Most centres now use plastic tracheostomy tubes in infants. The tube designed by Aberdeen (1965) has been very satisfactory (Stool, Campbell \& Johnson, 1968; Talbert \& Haller, 1968). Tubes of this type adapt to the individual infant's trachea and therefore do not cause excessive pressure at any point. Silver cannulae with a removable inner tube are unnecessary. The purpose of the inner tube is to enable a blocked tube to be cleared. Humidification, which is essential for the reasons already described, eliminates the need for an inner tube. Furthermore, a rigid tube must always produce pressure at certain points as it cannot adapt to the individual trachea. This pressure may occur on a tracheal ring with consequent erosion of it and collapse of the trachea leading to decannulation difficulties. Pressure may also occur at the tip of the tube causing ulceration of the wall of the trachea.

An important point about tracheostomy tubes in infants and young children is that an inflatable cuff is unnecessary. By putting in the largest tracheo- stomy tube that will comfortably fit the trachea, one has a sufficiently leak-proof fit to be satisfactory clinically, thereby enabling quite high inflating pressures to be used. There is ample evidence now that inflatable cuffs increase the incidence of trachea trauma (Stiles, 1965: Davidson et al., 1971).

Management. As in prolonged endotracheal intubation, continuous nursing supervision and good humidification are essential. If the patient shows signs of respiratory embarrassment after the tracheostomy tube is in place one must exclude the possibility of a pneumothorax.

Changing the tracheostomy tube. If humidification is adequate it is seldom necessary to change the tube. It may be changed once per week to enable complete toilet to be done to the stoma. In the first 3 days it should not be changed outside the operating theatre as it may be extremely difficult to replace it before a track has formed.

Prevention of infection. The danger of infection is greatest in premature babies. If a tracheostomy is performed on such an infant the greatest care must be taken to avoid infection, e.g. sterile gloves should be worn when inserting the sterile suction catheter into the trachea. In infants even a few weeks old there is much less danger from this source. Many infants of this age already carry pathogens such as $P$. aeruginosa or coagulase-positive staphylococci in their nose, throat or rectum. It is almost inevitable that these organisms will be isolated, within a few days, from the tracheal aspirate. Such patients do not show signs of clinical infection and it is not necessary to treat them with antibiotics in such circumstances. A 'no-touch' technique should be used when introducing the sterile catheter to aspirate the trachea of these older infants, i.e. the part of the catheter introduced into the trachea need not be handled.

Decannulation. If tracheostomy has been performed as described and if a plastic tracheostomy tube has been used, then, provided the upper airway was normal before the tracheostomy was done, one does not anticipate difficulty in decannulation. Damage to the first ring of the trachea is the commonest cause of decannulation difficulty. It is of course essential, before attempting decannulation, to ensure that the infant is in a satisfactory condition, i.e. secretions should be minimal and cardiac failure or other causes of respiratory difficulty should have been eliminated.

Young infants often do not breathe normally after decannulation. This may be because coordination between the larynx and respiration is lost. For example, the cords may remain in apposition on inspiration and as a result the infant struggles for air and becomes cyanosed as the stoma closes. One method of attempting to overcome this problem is to 
sedate the infant fairly heavily with morphine $(0 \cdot 2$ $\mathrm{mg} / \mathrm{kg}$ ) before decannulation. The sedated infant usually continues to sleep, the stoma closes gradually and more and more air enters via the normal airway. When the sedation has worn off respiration is usually well established by the normal route and the stoma may be almost closed.

There is one important point to note. If in spite of sedation an infant shows signs of obstructed respiration as the stoma closes then one must assume that there is an organic obstruction until proved otherwise. The tracheostomy tube should be replaced and, under full general anaesthesia, an endoscopy should be performed to exclude such things as granulations or a mucosal flap at the site of the stoma.

The choice of airway. Many infants and young children in several centres have been managed by either prolonged endotracheal intubation or by tracheostomy. The supervision and the general techniques are similar for both methods and simple mistakes can easily be fatal in these ill patients, whichever method is used.

An endotracheal tube seems to be more difficult to manage from the nursing point of view and is less pleasant for the patient. The danger of erosion of the nose is a constant anxiety and becomes more difficult to avoid as time goes on. The serious complication of sub-glottic stenosis although probably related mainly to the use of too large a tube may also have some relation to the duration of intubation. It would seem therefore that the case for intubation becomes weaker the longer the tube is required.

A tracheostomy does, of course, involve a small operation and a scar on the neck. In a few patients there will be decannulation difficulties if the operation has been performed at too high a level and in a few patients granulations may form in the trachea at the stoma.

If a patient requires an artificial airway for a few days it seems reasonable to use an endotracheal tube. If at the end of this time the patient is not improving it is probably best to do a tracheostomy at that stage.

Both endotracheal intubation and tracheostomy have made considerable contributions to the care of ill patients and each method should be regarded as complementing the other rather than excluding it.

\section{The role of mechanical ventilation in paediatric surgery}

Although the causes of respiratory insufficiency are many and varied, mechanical ventilation is always in a supportive role while the basic cause is being treated. It follows that if the basic cause is not successfully treated then mechanical ventilation will merely delay the probable fatal outcome.

In the author's experience, the best results asso- ciated with mechanical ventilation have been in infants and children undergoing cardiac surgery. In this group of patients we are dealing with respiratory insufficiency secondary to cardiac failure or ventilation/perfusion upsets of a transitory nature in the lung following cardiac surgery. It is logical to give mechanical support in such conditions and, provided the surgery itself has been successful, then artificial ventilation is usually necessary for a few days only leading to full recovery.

In neonatal surgery of the oesophagus, repair of diaphragmatic hernia or surgery of the intestines, mechanical ventilation when required is less rewarding for various reasons.

(a) In an otherwise normal infant undergoing oesophageal or intestinal surgery, mechanical ventilation is rarely necessary immediately postoperatively. If it does become necessary a few days later, it will probably be the result of serious complications such as severe peritonitis from a leaking intestinal anastomosis or pneumonia secondary to an extensive leak from the oesophageal anastomosis. Early and successful surgery may be necessary to avoid a fatal outcome from these complications. In these circumstances mechanical ventilation will be in a supportive role and survival will depend on the success of the surgical management.

(b) In congenital diaphragmatic hernia, artificial ventilation is often necessary before and after surgery. In such infants, requiring artificial ventilation within a few hours of birth, results are poor. The contralateral lung may be hypoplastic in addition to severe hypoplasia of the lung on the affected side. In addition, there are probably severe ventilation/perfusion upsets in the lung as these infants may remain seriously desaturated even when ventilated with $100 \%$ oxygen.

(c) Some neonates requiring surgery may be premature and therefore may require artificial ventilation immediately postoperatively. Such patients may have respiratory distress syndrome associated with their prematurity. Results will vary in this group depending on the degree of prematurity, the severity of the RDS and the extent of the surgery.

It is the policy in some centres to ventilate a high proportion of neonates in the immediate postoperative period. This will give a much higher percentage survival in the total results and must be remembered when interpreting those results.

In conclusion, it can be said that in the total field of paediatric surgery mechanical ventilation has an important supportive role to play. This supportive role must be stressed otherwise too much may be expected from it.

The nature of the underlying condition necessitating artificial ventilation will usually determine the outcome. 


\section{References}

AberdeEn, E. (1965) Tracheostomy and tracheostomy care in infants. Proceedings of the Royal Society of Medicine, 58, 900 .

AberdeEn, E. (1968) Tracheostomy in infants. In: Operative Surgery (Ed. by C. Rob and R. Smith), 2nd edn. Butterworth, London.

Barnes, N.D., Glover, W.J., Hull, D. \& Milner, A.D. (1969) Effects of prolonged positive pressure ventilation in infancy. Lancet, ii, 1096.

BaUm, J.D. \& Tizard, J.P.M. (1970) Retrolental fibroplasia: Management of oxygen therapy. British Medical Bulletin. 26, 171.

Caldweli, P.R.B., Lee, W.L., Schildkraut, H.S. \& ARChibald, E.R. (1966) Changes in lung volume, diffusing capacity and blood gases in men breathing oxygen. Journal of Applied Physiology, 21, 1477.

Cross, K.W. (1965) Handbook of Physiology, Section 3, Respiration, Vol. II. American Physiological Society, Washington, D.C.

Dalhamn, T. (1956) Mucous flow and ciliary activity in the trachea of healthy rats and exposed to respiratory irritant gases. Acta physiologica Scandinavica, 36, Suppl. 123.

Davidson, I.A., Cruickshank, A.N., Duthie, W.H., Bargh, W. \& DuncaN, J.G. (1971) Tracheal lesions following tracheostomy. Proceedings of the Royal Society of Medicine, 64, 886.

FEARON, B. (1962) Acute laryngotracheobronchitis in infancy and childhood. Pediatric Clinics of North America, 9, 1095.

Fenstermaker, D.E. (19\%) A review of ultrasonic nebulization. In: Prolonged tracheal intubation (Ed. by J. B. Stetson). International Anesthesiology Clinics, 8, 949.

Glover, W.J. (1965) Mechanical ventilation in respiratory insufficiency in infants. Proceedings of the Roval Society of Medicine, 58, 902.

Glover, W.J. (19\%0) Tracheostomy in the intensive care of infants. In: Progress in Anaesthesiology, International Congress Series No. 200, p. 451. Excerpta Medica Foundation, Amsterdam.
Guess, W.L. (1970) Tissue testing of polymers. In: Prolonged tracheal intubation (Ed. by J. B. Stetson). International Anaesthesiology Clinics, 8, 787.

Guess, W.L. \& Stetson, J.B. (1968) Tissue reactions to organotin-stabilized polyvinyl chloride (PVC) catheters. Journal of the American Medical Association, 204, 580.

Hogg, J.C., Williams, J., Richardson, J.B., Macklem, P.T. \& Thurlbeck, W.M. (1970) Age as a factor in the distribution of lower-airway conductance and in the pathologic anatomy of obstructive lung disease. New England Journal of Medicine, 282, 1283.

Holinger, P.H., Brown, W.T. \& Maurizi, D.G. (1965) Tracheostomy in the newborn. American Journal of Surgery, 109, 771.

Modell, J.H., Moya, F., Ruiz, B.C., Showers, A.V. \& NewBy, E.J. (1968) Blood gas and electrolyte determinations during exposure to ultrasonic nebulized aerosols. British Journal of Anaesthesia, 40, 20.

Moffet, H.L. \& Allan, D. (1967) Colonization of infants exposed to bacterially contaminated mists. American Journal of Diseases of Children, 114, 21.

Stiles, P.J. (1965) Tracheal lesions after tracheostomy. Thorax, 20, 517.

Stocks, J. (1970) Prolonged nasal intubation in paediatric intensive care. In: Progress in Anaesthesiology, International Congress Series No. 200, p. 447. Excerpta Medica Foundation, Amsterdam.

Stool, S.E., Campbell, J.R. \& Johnson, D.G. (1968) Tracheostomy in children: The use of plastic tubes. Journal of Pediatric Surgery, 3, 402.

TAlberT, J.L. \& Haller, J.A. (1968) Improved silastic tracheostomy tubes for infants and young children. Journal of Pediatric Surgery, 3, 408.

WatTs, J. McK. (1963) Tracheostomy in modern practice. British Journal of Surgery, 50, 954.

Wolfsdorf, J., Swift, D.L. \& Avery, M.E. (1969) Mist therapy reconsidered; An evaluation of the respiratory deposition of labelled water aerosols produced by jet and ultrasonic nebulizers. Pediatrics, 43, 799. 